\title{
Traumatic Brain Injury in an Aging Population
}

\author{
Nino Stocchetti, Rosalia Paternò,, Giuseppe Citerio,, Luigi Beretta, ${ }^{3}$ and Angelo Colombo ${ }^{1}$
}

\begin{abstract}
The epidemiology of traumatic brain injury (TBI) is changing in several Western countries, with an increasing proportion of elderly TBI patients admitted to the intensive care unit (ICU). We describe a series of 1366 adult patients admitted to three neuro-ICUs in which $44 \%$ of cases were 50 years of age or older. The health status before trauma (rated using the APACHE score) was worse in older patients. In all 604 patients had emergency removal of intracranial masses, with extradural hematomas more frequent in young cases and subdural hematomas more frequent in older patients. Outcomes were classified according to the Glasgow Outcome Scale (GOS) 6 months post-trauma, as favorable (GOS score 4-5), or unfavorable (GOS score 1-3). Favorable outcomes were achieved by $50 \%$ of patients, but the proportions of unfavorable outcomes rose with age. Mortality was the main cause of unfavorable outcomes 6 months after injury in older patients. Logistic regression analysis indicates that several parameters independently contributed to outcome, including the motor component of the Glasgow Coma Scale (GCS), pupils, CT findings, and early hypotension. Additionally, the odds ratios were very high for age and health status before TBI. Patients admitted to the ICU are increasingly older, have co-morbidities, and have specific types of intracranial lesions. Early rescue, surgical treatment, and intensive care of these patients may produce excellent results up to the age of 59 years, with favorable outcomes still possible for $39 \%$ of cases aged 60-69 years, without an excessive burden of severely disabled patients.
\end{abstract}

Key words: adult brain injury; age; geriatric brain injury; outcome measures

\section{Introduction}

$\mathbf{T}$ RAUMATIC BRAIN INJURY (TBI) is a major cause of morbidity and mortality. Its epidemiology, however, is changing. In the emerging economies such as China and India, where motorized travel is quickly growing, TBI is also increasing, as road traffic accidents (RTA) become more frequent, whereas in most Western countries its incidence is falling. One reason is that successful RTA prevention is leading to fewer accidents and less TBI among young people (World Health Organization, 2010). However, the aging of the population has shifted the epidemiology of TBI toward more frequent falls at home, mainly involving the elderly.

The importance of age as a prognostic factor after TBI has long been recognized. More than 30 years ago the first prognostic models indicated age as an independent outcome predictor (Heiden et al., 1979), and this has been confirmed by more recent figures from the IMPACT group (Mushkudiani et al., 2007).

Several factors can contribute to worse outcomes in the elderly. The mechanism of injury is different across age groups. While the young are often victims of RTA, older people more often fall at home, with a higher incidence of subdural hematoma, which is associated with a worse outcome (Howard et al., 1989; Thompson et al., 2006).

Age is also associated with co-morbidities, such as diabetes, hypertension, and chronic renal failure, which may worsen outcomes. Also, a high percentage of older people use medications (e.g., anticoagulants and/or anti-platelets) which may worsen cerebral damage after trauma. The aged brain may be more vulnerable to TBI, with less plasticity and repair after injury (Kovacs, 2005). In addition, older people often present with alterations in cognition, memory, mood, and motor function (Glorioso and Sibille, 2011), which can hamper rehabilitation after TBI. Finally, the widely held view that outcomes after TBI in the elderly are bad may induce less aggressive treatment, both surgical and medical, for these cases.

In general, however, the outcome after TBI seems to have improved with time (Lu et al., 2005; Stein et al., 2010), and this progress may possibly include older patients who were previously classified as almost invariably unsalvageable.

\footnotetext{
${ }^{1}$ University of Milan, and NeuroIntensive Care Unit, Fondazione IRCCS Cà Granda Ospedale Maggiore Policlinico, Milano, Italy.

${ }^{2}$ NeuroIntensive Care Unit, Ospedale San Gerardo, Monza, Italy.

${ }^{3}$ NeuroIntensive Care Unit, Ospedale San Raffaele, Milano, Italy.
} 
The main aims of this article are: (1) to describe the principal clinical features of a consecutive series of adult TBI cases including a number of elderly patients, focusing on differences between different age groups, and (2) to verify the main determinants of 6-month outcomes.

\section{Methods}

\section{Inclusion/exclusion criteria}

Patients admitted consecutively to three neurosurgical ICUs (belonging to Ospedale Maggiore Policlinico and Ospedale San Raffaele, both in Milan, and the third to Ospedale San Gerardo, Monza, Italy) from January 1, 1997 to December 31, 2007 were prospectively studied. The inclusion criteria were: (1) admission because of head trauma, with or without extracranial injuries; (2) brain injury severity requiring admission to an ICU; (3) time from trauma to arrival at the ICU < 24h; and (4) age over 18 years.

Patients whose severity was probably overestimated on admission because of sedation and/or muscle relaxants were identified according to published criteria (Stocchetti et al., 2004), and defined as mistakenly severe (MS); they were excluded from further analysis.

\section{Data collection}

The health status before the trauma was rated using a simple score (Acute Physiology, Age, and Chronic Health Evaluation; APACHE), classifying patients under four headings: (A) prior good health, no functional limitations; (B) mild to moderate limitation of activity because of a chronic medical problem; (C) chronic disease causing serious but not incapacitating restriction of activity; and (D) severe restriction of activity because of disease (Knaus et al., 1981).

Before arrival at the neurotrauma center hypoxia was defined as arterial saturation $<90 \%$ and/or blood gas analysis with $\mathrm{PAO}_{2}<60 \mathrm{~mm} \mathrm{Hg}$. When saturation or blood gas analysis was not available but the patient appeared cyanotic, and/ or had airway obstruction, hypoxia was recorded as suspected. Hypotension was defined as systolic pressure $<95 \mathrm{~mm} \mathrm{Hg}$; when arterial pressure was not measured but the patient had weak or not detectable arterial pulse, hypotension was coded as suspected.

Repeated CT scans were done on all patients. The scan that was judged most clinically important (i.e., the one that showed the worst brain damage) was entered into the database. We applied a modified version of the Marshall classification, grouping all mass lesions, evacuated and not evacuated, in one class (Marshall et al., 1991). Extradural and subdural hematomas, the status of basal cisterns, the amount of midline shift, and the presence of subarachnoid hemorrhage, were coded as separate items.

Patients were managed according to protocols published previously (Stocchetti et al., 2008). All data were entered into a computerized database described elsewhere (Beretta et al., 2003). Outcome was assessed by personal interview or by phone 6 months after injury, using a structured questionnaire for the Glasgow Outcome Scale (GOS; Wilson et al., 1998).

The ethics committee of the hospital where the database was developed (Ospedale Policlinico, Milan, Italy) gave approval for the data to be used for research and publication. Because the patients were unconscious when admitted, rela- tives or next of kin were informed that clinical data were being rendered anonymous and stored for research.

\section{Statistical analysis}

The first part of the analysis was descriptive. Mean and standard deviation were calculated for continuous data with normal distribution; median and range were reported when the distribution was not normal or in case of categorical data.

Differences between age groups for various parameters were assessed using analysis of variance (ANOVA), or chisquare testing when appropriate. A $p$ value $<0.05$ was considered significant. Multivariate analysis was done by logistic regression. Using the method of maximum likelihood we calculated coefficients and corresponding odds ratios (OR) with $95 \%$ confidence intervals (CI). As a dependent variable, outcome was split into favorable (good recovery and moderate disability) or all other categories. As independent variables, we entered into the model the parameters used in the IMPACT project (age, GCS motor score, pupils, the Marshall CT classification, and traumatic subarachnoid hemorrhage, hypoxia, or hypotension in the pre-hospital phase; Murray et al., 2007). All variables were dichotomized; age, in decades, was split at 60 years. Pupils were coded as bilaterally normal or unilaterally/bilaterally dilated. Then the health status before the trauma was added and coefficients were recalculated.

The odds ratios were calculated so that a value greater than 1 indicated a higher risk of a poor outcome relative to the reference category. The ratio of the difference to the reduced negative log-likelihood values (indicated as R-square) was calculated. Finally, a lack-of-fit test was run to assess whether there was enough information using the variables entered in the model.

The data were analyzed using Data Desk v.6 (Data Description, Ithaca, NY) and JMP 8.02 (SAS Institute Inc., Cary, NC) statistical software.

\section{Results}

The database included 1478 patients older than 18 years, from January 1997 to December 2007; 112 fulfilled the criteria for MS, leaving 1366 cases suitable for analysis. The median age was 45 years (range 19-94 years), and 44\% were 50 years old or older. Patients were divided into seven age groups, as indicated in Table 1.

APACHE data were available for 1356 cases. Older patients had a higher proportion of cases with severe limitations. The distribution of the APACHE score among the age groups was significantly different $(p \leq 0.0001)$. There were no differences in the demographic features between the three centers who participated in the study.

Before arrival at the neurotrauma center, 19\% of cases had documented hypoxia and $12 \%$ had suspected hypoxia. Arterial hypotension was documented in 19\%, and suspected in $4 \%$. A total of 174 patients required emergency surgery for extracranial lesions.

Reliable GCS scores after stabilization were available for 1350 patients. The motor component of the GCS was 1-2 (no motor response or extension) in 27\%, and 3-4 (abnormal flexion or withdrawal to pain) in $20 \%$; 471 patients (35\%) localized to pain, and $243(18 \%)$ could obey commands. Anisocoria was detected in $24 \%$ and bilateral dilation in $10 \%$, while pupils were normal in $66 \%$. 
Table 1. APaCHE Classification of Health Status Preceding Trauma

\begin{tabular}{|c|c|c|c|c|c|c|c|c|}
\hline Age (years) & $\begin{array}{l}19-29 \\
\text { n }(\%)\end{array}$ & $\begin{array}{l}30-39 \\
\text { n (\%) }\end{array}$ & $\begin{array}{l}40-49 \\
\text { n }(\%)\end{array}$ & $\begin{array}{l}50-59 \\
\text { n (\%) }\end{array}$ & $\begin{array}{l}60-69 \\
\text { n (\%) }\end{array}$ & $\begin{array}{l}70-79 \\
\text { n (\%) }\end{array}$ & $\begin{array}{c}\geq 80 \\
\mathrm{n}(\%)\end{array}$ & $\begin{array}{c}\text { Total } \\
\mathrm{n}(\%)\end{array}$ \\
\hline Patients & $356(26 \%)$ & $241(18 \%)$ & $166(12 \%)$ & $168(12 \%)$ & $164(12 \%)$ & $179(13 \%)$ & $92(7 \%)$ & 1366 \\
\hline Female & 68 (19\%) & $49(20 \%)$ & 42 (25\%) & $39(23 \%)$ & $37(22 \%)$ & $72(40 \%)$ & 46 (50\%) & $353(26 \%)$ \\
\hline Male/female ratio & $4: 23$ & $3: 92$ & $2: 95$ & $3: 31$ & $3: 43$ & $1: 49$ & 1 & $2: 87$ \\
\hline APACHE A & $332(93 \%)$ & $218(91 \%)$ & $131(79 \%)$ & $118(71 \%)$ & $85(52 \%)$ & $50(28 \%)$ & $27(30 \%)$ & $961(71 \%)$ \\
\hline APACHE B & $18(5 \%)$ & $17(7 \%)$ & $29(18 \%)$ & $40(24 \%)$ & $63(39 \%)$ & $96(54 \%)$ & $39(43 \%)$ & $302(22 \%)$ \\
\hline APACHE C & $2(1 \%)$ & $4(2 \%)$ & $4(2 \%)$ & $5(3 \%)$ & $11(7 \%)$ & $25(14 \%)$ & $22(25 \%)$ & $73(5 \%)$ \\
\hline APACHE D & $1(1 \%)$ & $0(0 \%)$ & $2(1 \%)$ & $4(2 \%)$ & $4(2 \%)$ & $7(4 \%)$ & $2(2 \%)$ & $20(2 \%)$ \\
\hline
\end{tabular}

M/F ratio: number of male for every female for each column.

APACHE, Acute Physiology, Age, and Chronic Health Evaluation.

Emergency removal (during the first $24 \mathrm{~h}$ ) of an intracranial mass was needed in 604 patients: extradural hematomas were removed in 206 cases, subdural in 367.

CT scan findings are summarized in Table 2. Extradural hematomas were more frequent in young patients and subdural hematomas were predominant in older patients (Fig. 1).

\section{General management and length of ICU stay}

The median length of stay in the ICU was 7 days (range 1-58 days). In the subgroup of patients who did not die in the ICU, the median length of stay in the ICU was 10 days. All patients submitted to sequential clinical neurologic monitoring, ECG monitoring, pulse oximetry, and temperature monitoring. Standard care included invasive arterial monitoring, artificial ventilation, blood gas analysis, blood and urine chemistry, correction of glucose and electrolyte disorders, and nutrition (if necessary artificial enteral nutrition was started on the second day after injury). Intracranial pressure was monitored in 901 patients (66\% of the total).

\section{Outcome}

Outcome data were available for 1273 cases. Outcomes by dichotomous GOS score in the different age groups are shown in Table 3. In all, 302 patients died in the ICU; 155 of the survivors were transferred from the admitting ICU to another ICU, 178 to a step-down unit, and 731 to a regular ward. There were no differences between the proportions of favorable/ unfavorable cases in the three centers.
As detailed in the methods section, the relationships between outcome and several variables were explored in steps. First, univariate analysis was done, as reported in Table 4 . In a second step, multivariable logistic regression was calculated using parameters reported by the IMPACT group; the data showed a non-significant contribution of pre-hospital hypoxia $(p=0.3)$. This variable was therefore excluded from further analysis. $\mathrm{R}^{2}$ was 0.305 , the lack-of-fit chi-square was 0.09 (not significant), and receiver operating curve (ROC) analysis gave an area under the curve of 0.847 .

In a third step the health status preceding TBI was included in the model (Table 5). The model included 1163 patients and worked adequately; $\mathrm{R}^{2}$ was 0.3193 , the lack-of-fit chi-square was 0.1256 (not significant), and ROC analysis gave an area under the curve of 0.855 .

\section{Discussion}

\section{Main findings}

The first aim of the study was to describe the main clinical features of a consecutive series of adult TBI patients admitted to the ICU, with special attention paid to elderly patients. The median age of this series (45 years), the substantial proportion of cases aged 50 years or more $(44 \%)$, and the fact that 1 patient in 5 was 70 years of age or older confirm that the epidemiology of TBI is changing in the Western world, as previously reported (Maas et al., 2008; Thompson et al., 2006; World Health Organization, 2010).

Table 2. Main CT Scan Data

\begin{tabular}{|c|c|c|c|c|c|c|c|c|}
\hline Age (years) & $\begin{array}{l}19-29 \\
\text { n (\%) }\end{array}$ & $\begin{array}{l}30-39 \\
\text { n (\%) }\end{array}$ & $\begin{array}{l}40-49 \\
\text { n (\%) }\end{array}$ & $\begin{array}{l}50-59 \\
\text { n (\%) }\end{array}$ & $\begin{array}{l}60-69 \\
\text { n (\%) }\end{array}$ & $\begin{array}{l}70-79 \\
\text { n (\%) }\end{array}$ & $\begin{array}{c}\geq 80 \\
\mathrm{n}(\%)\end{array}$ & $\begin{array}{c}\text { Total } \\
\text { n ( }(\%)\end{array}$ \\
\hline Diffuse injury I & $36(11 \%)$ & $15(6 \%)$ & $13(8 \%)$ & $7(4 \%)$ & $10(6 \%)$ & $5(3 \%)$ & $2(2 \%)$ & $88(6 \%)$ \\
\hline Diffuse injury II & $115(32 \%)$ & $81(34 \%)$ & $46(27 \%)$ & $54(32 \%)$ & $37(23 \%)$ & $35(20 \%)$ & $16(17 \%)$ & $384(28 \%)$ \\
\hline Diffuse injury III & $51(14 \%)$ & $32(13 \%)$ & $18(11 \%)$ & $8(5 \%)$ & $12(8 \%)$ & $5(3 \%)$ & $7(8 \%)$ & $133(10 \%)$ \\
\hline Diffuse injury IV & $12(3 \%)$ & $6(2 \%)$ & $6(4 \%)$ & $4(2 \%)$ & $3(1 \%)$ & $4(2 \%)$ & $0(0 \%)$ & $35(3 \%)$ \\
\hline Mass lesions & $142(40 \%)$ & $107(45 \%)$ & $83(50 \%)$ & $95(57 \%)$ & $102(62 \%)$ & $130(72 \%)$ & $67(73 \%)$ & $726(53 \%)$ \\
\hline $\mathrm{tSAH}$ & $186(52 \%)$ & $130(54 \%)$ & $101(62 \%)$ & $112(67 \%)$ & $110(67 \%)$ & $118(66 \%)$ & $52(56 \%)$ & $809(59 \%)$ \\
\hline Cisterns compressed/absent & $160(45 \%)$ & $101(42 \%)$ & $77(47 \%)$ & $80(48 \%)$ & $77(47 \%)$ & $90(50 \%)$ & $50(54 \%)$ & $635(46 \%)$ \\
\hline Midline shift $>5 \mathrm{~mm}$ & $86(24 \%)$ & $63(26 \%)$ & $58(35 \%)$ & $65(39 \%)$ & $67(41 \%)$ & $93(53 \%)$ & $53(58 \%)$ & $485(36 \%)$ \\
\hline
\end{tabular}

Computed tomography (CT) scans have been coded according to a modified version of the Marshall classification: non-evacuated and evacuated mass lesions were grouped in the same class.

The last three rows report the presence of traumatic subarachnoid hemorrhage ( $\mathrm{tSAH})$, the status of the mesencephalic cisterns, and the midline shift. 


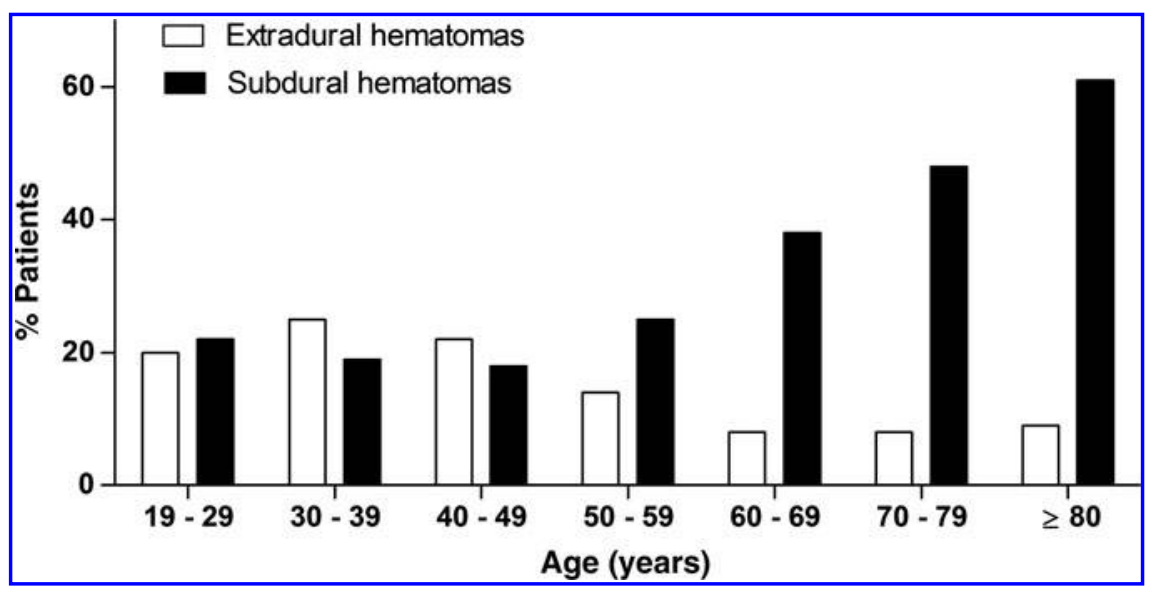

FIG. 1. Proportion of patients with subdural or extradural hematomas in different age groups. The distribution is statistically different $(\mathrm{p}<0.0001)$.

Patients were mainly male, as is common with TBI. However, there was a higher proportion $(43 \%)$ of females in the group older than 69 years. This may depend on different mechanisms of injury, which unfortunately were not collected in our database. Traffic accidents, which account for the majority of TBI in young people, mainly involve males, while falls at home, more common in the elderly, involve both sexes. This might also be explained by the significant proportion of females in the oldest part of the population. In the area served by our hospitals the percentage of females in the general population older than 69 years is $62 \%$ (ISTAT, 2002).

While younger patients were typically healthy, a larger proportion of older patients prior to TBI had chronic diseases causing serious or severe incapacitating restriction of activity.

The three centers contributing to the database serve as referring neurosurgical facilities for a large network of hospitals without neurosurgery. This implies that patients with a surgical indication are concentrated in these centers, which may help explain the high proportion of neurosurgical cases (44\%). Subdural hematomas became more frequent than extradural hematomas after the age of 50 years.

Seven percent of cases were lost to follow-up, most of them immigrants who are hard to track after hospital discharge. Favorable outcomes were recorded for $50 \%$, a noteworthy achievement considering two aspects: the relatively high median age and the previous exclusion of MS cases (Stocchetti et al., 2004). By definition, patients who appear to be severe because of sedation or muscle relaxants may achieve very good results, thus making the final pooled results appear to be more favorable. Of course, exclusion of such patients can worsen the overall outcome picture.

The proportion of unfavorable outcomes in our series rose with age, as expected. Relatively few patients were left in a vegetative state, and the figures remained constant over the age groups; however, severe disability was $12 \%$ for those less than 60 years of age, but $18 \%$ for older patients. Mortality was the main cause of unfavorable outcomes 6 months after injury in older patients. Few patients younger than 50 years of age discharged alive from an ICU die in the following months, whereas the proportion of persons older than 50 who die after leaving the ICU is much higher. The causes of these deaths after ICU discharge cannot be identified from the database, and only hypotheses can be offered. It is likely that the combination of existing co-morbidities (e.g., diabetes and cardiac failure), with the long-term consequences of TBI (e.g., bedridden, inadequate nutrition, or recurrent infections), contributed to late mortality.

Clinical experience and univariate analysis suggest that several parameters may be associated with unfavorable outcome, including the biological status before injury (age and

Table 3. Six-Month Outcome by the Glasgow Outcome Scale Score

\begin{tabular}{|c|c|c|c|c|c|c|c|c|}
\hline Age (years) & $19-29$ & $30-39$ & $40-49$ & $50-59$ & $60-69$ & $70-79$ & $\geq 80$ & All cases \\
\hline Died & $63(19 \%)$ & $44(20 \%)$ & $38(24 \%)$ & $47(31 \%)$ & $57(37 \%)$ & $100(59 \%)$ & $67(79 \%)$ & $416(33 \%)$ \\
\hline Died in the ICU & $61(18 \%)$ & $40(18 \%)$ & $33(21 \%)$ & $30(19 \%)$ & $41(26 \%)$ & $61(36 \%)$ & $36(42 \%)$ & $302(24 \%)$ \\
\hline Vegetative & $11(3 \%)$ & $10(4 \%)$ & $6(4 \%)$ & $5(3 \%)$ & $5(3 \%)$ & $2(1 \%)$ & $2(2 \%)$ & $41(3 \%)$ \\
\hline Severe disability & $34(10 \%)$ & $28(13 \%)$ & $29(18 \%)$ & $16(10 \%)$ & $32(21 \%)$ & $35(21 \%)$ & $5(6 \%)$ & $179(14 \%)$ \\
\hline Moderate disability & $66(20 \%)$ & $47(22 \%)$ & $31(20 \%)$ & $37(24 \%)$ & $16(10 \%)$ & $12(7 \%)$ & $4(5 \%)$ & $213(17 \%)$ \\
\hline Good recovery & $162(48 \%)$ & 89 (41\%) & $53(34 \%)$ & 49 (32\%) & $44(29 \%)$ & $20(12 \%)$ & $7(8 \%)$ & $424(33 \%)$ \\
\hline Unfavorable & $108(32 \%)$ & $82(37 \%)$ & $73(46 \%)$ & $68(44 \%)$ & $94(61 \%)$ & $137(81 \%)$ & $74(87 \%)$ & $636(50 \%)$ \\
\hline Favorable & $228(68 \%)$ & $136(63 \%)$ & $84(54 \%)$ & $86(56 \%)$ & $60(39 \%)$ & $32(19 \%)$ & $11(13 \%)$ & $637(50 \%)$ \\
\hline Total & 336 & 218 & 157 & 154 & 154 & 169 & 85 & 1273 \\
\hline
\end{tabular}

Percentages are calculated by column. Favorable includes good recovery and moderate disability; unfavorable indicates death, vegetative state, or severe disability.

ICU, intensive care unit. 
Table 4. Univariate Analysis of Potential Outcome Predictors

\begin{tabular}{|c|c|c|c|}
\hline & $\begin{array}{l}\text { Favorable } \\
\text { outcome } \\
\text { n (\%) }\end{array}$ & $\begin{array}{l}\text { Unfavorable } \\
\text { outcome } \\
\text { n }(\%)\end{array}$ & $\begin{array}{c}\mathrm{p} \text { Value } \\
\mathrm{n}(\%)\end{array}$ \\
\hline APACHE cases classified as C and D & $11(12 \%)$ & $78(88 \%)$ & $\leq 0.0001$ \\
\hline Age $>59$ years & $103(25 \%)$ & $305(75 \%)$ & $\leq 0.0001$ \\
\hline Hypoxia documented or suspected & $146(38 \%)$ & $234(62 \%)$ & $\leq 0.0001$ \\
\hline Hypotension documented or suspected & $104(35 \%)$ & $192(65 \%)$ & $\leq 0.0001$ \\
\hline Motor component of GCS $<4$ & $105(23 \%)$ & $356(77 \%)$ & $\leq 0.0001$ \\
\hline Pupils (one or two dilated) & $95(23 \%)$ & $314(77 \%)$ & $\leq 0.0001$ \\
\hline Marshall CT scan classification: diffuse injury greater than II or mass lesion & $333(39 \%)$ & $512(61 \%)$ & $\leq 0.0001$ \\
\hline $\mathrm{tSAH}$ & $324(43 \%)$ & $425(57 \%)$ & $\leq 0.0001$ \\
\hline
\end{tabular}

tSAH, traumatic subarachnoid hemorrhage; GCS, Glasgow Coma Scale; APACHE, Acute Physiology, Age, and Chronic Health Evaluation; $\mathrm{CT}$, computed tomography.

prior health status), hypoxia, and hypotension during the rescue phase, the severity of neurological damage (pupils and the motor component of the GCS), and intracranial lesions (CT scan data). When these parameters were entered into a multivariable analysis, all but hypoxia appeared significant.

Logistic regression indicates that several parameters independently contribute to outcome. In particular, the odds ratios are very high for age and health status before TBI: the probability of an unfavorable outcome for persons older than 59 years is six times that for younger patients. Pre-existing diseases cause approximately the same probability of unfavorable outcome.

Older patients more frequently have a worse health status, and it is extremely important to distinguish the relative importance of age per se and the diseases they already have. From logistic analysis, the pre-existing health status seems to be an important independent predictor; however, numbers in this series were low for this specific item; only 22 patients younger than 60 years were in the worst APACHE categories.

Other predictors are confirmed as important too, particularly the neurological findings (pupils and motor score) assessed early on admission, after oxygenation and arterial pressure were stabilized.

\section{The prognostic importance of age in the elderly}

The strong prognostic importance of age has been amply acknowledged in the TBI literature. Early reports indicated unfavorable results in older patients. One even showed that patients older than 30 years had overwhelming mortality (75-80\%), with no survivors at 1 year for those older than 60 years (Heiden et al., 1979). In another study mortality was $53 \%$ in patients older than 29 years and $81 \%$ after 60 years (Teasdale and Jennett, 1976). In a comparison of old and young patients with subdural hematomas, mortality rates were more than four times higher in older patients, and no case with a GCS score $<13$ had a functional outcome (Howard et al., 1989). The prognostic tree method identified two ages as prognostic thresholds associated with worse outcome: older than 26 years and older than 60 years (Choi et al., 1991).

Subsequent data based on thousands of cases have confirmed age as a strong outcome predictor (Hukkelhoven et al., 2003; Mushkudiani et al., 2007). This last analysis in particular found a continuous effect of age on outcome, while our analysis was based on age groups. Fortunately, in more recent series outcome seems to be less hopeless than it was decades ago (Brazinova et al., 2010), and the threshold for unacceptable results appears to have been extended considerably (Boruas et al., 2007).

Age is associated with co-morbidities, since a large proportion of old patients suffer from diabetes, hypertension, and cerebrovascular disease (Thompson et al., 2006). Medications such as anticoagulants and antiplatelet agents, frequently used in older persons, may worsen cerebral damage after trauma (Woolcott et al., 2009). It remains to be established whether the worse outcome after TBI in the elderly is due

Table 5. Logistic Regression Analysis for the Probability of 6-Month Unfavorable Outcome

\begin{tabular}{lccr}
\hline Variable & $\begin{array}{c}\text { Estimated logistic } \\
\text { regression coefficient }(\beta)\end{array}$ & p Value & $\begin{array}{c}\text { Odds ratio } \\
\text { (95\% confidence interval) }\end{array}$ \\
\hline Intercept & -1.585 & $<0.0001$ & $6.05(4.332-8.527)$ \\
Age $>59$ years & 0.9 & $<0.0001$ & $4.634(3.279-6.584)$ \\
Motor component of GCS $<4$ & 0.766 & $<0.0001$ & $3.109(2.199-4.409)$ \\
Pupils: one or two dilated & 0.567 & $<0.0001$ & $1.945(1.370-2.773)$ \\
Hypotension documented or suspected & 0.332 & 0.0002 & $2.233(1.630-3.071)$ \\
Marshall CT scan classification: diffuse & 0.401 & $<0.0001$ & $1.622(1.202-2.194)$ \\
$\quad$ Injury greater than II or mass lesions & & 0.0016 & $5.901(2.894-13.192)$ \\
Traumatic subarachnoid hemorrhage & 0.241 & $<0.0001$ & 0.887
\end{tabular}

GCS, Glasgow Coma Scale; APACHE, Acute Physiology, Age, and Chronic Health Evaluation. 
mainly to concomitant diseases or to increased vulnerability of the central nervous system. Various series, however, indicate age as an independent predictor of worse outcome, after adjusting for covariates including co-existing diseases (Thompson et al., 2006; Tokutomi et al., 2008; Vollmer et al., 1991).

Mechanisms of injury change with age, with falls being increasingly important in the elderly population (Hukkelhoven et al., 2003; Maas et al., 2008). Concomitant medications such as hypnotics, antidepressants, and benzodiazepines, all increasingly used by the elderly, are clearly associated with falls (Woolcott et al., 2009). Older patients who have already experienced trauma are at increased risk of recurrent TBI (McGwin et al., 2001).

\section{Limitations of the study}

Our analysis is based on a low-cost database, which has several limitations (Beretta et al., 2007). The mechanisms of trauma were not recorded and the health status preceding trauma was documented with a simple but generic score, which does not give details of concurrent illnesses and medications.

There is also no information about the rehabilitation phase, which could be very important in the elderly, or on the causes of death after ICU discharge. Finally, the three ICUs contributing to this database act as referral centers to academic hospitals; this may influence the case mix and the results. The extension of our data to the general population should therefore be made with caution.

\section{Conclusions}

An aging population creates new challenges in TBI treatment. Patients admitted to the ICU are older, with COmorbidities, and with specific types of intracranial lesions. Early rescue, surgical treatment, and intensive care of these patients may produce excellent results up to the age of 59 years, with favorable outcomes still possible for $39 \%$ of cases aged 60-69 years, without an excessive burden of severely disabled patients. Late mortality after ICU discharge calls for further investigation.

\section{Author Disclosure Statement}

No competing financial interests exist.

\section{References}

Beretta, L., Aldrovandi, V., Grandi, E., Citerio, G., and Stocchetti, N. (2007). Improving the quality of data entry in a low-budget head injury database. Acta Neurochir. (Wien.) 149, 903-909.

Beretta, L., Grandi, E., Citerio, G., Cormio, M., and Stocchetti, N. (2003). Neuro-Link, an Italian Traumatic Coma Data Bank: what did we learn from the first 1000 patients and how can we do better? Minerva Anestesiol. 69, 223-226.

Bouras, T., Stranjalis, G., Korfias, S., Andrianakis, I., Pitaridis, M., and Sakas, D.E. (2007). Head injury mortality in a geriatric population: differentiating an "edge" age group with better potential for benefit than older poor-prognosis patients. J. Neurotrauma 24, 1355-1361.

Brazinova, A., Mauritz, W., Leitgeb, J., et al. (2010). Outcomes of patients with severe traumatic brain injury who have Glasgow
Coma Scale scores of 3 or 4 and are over 65 years old. J. Neurotrauma 27, 1549-1755.

Choi, S.C., Muizelaar, J.P., Barnes, T.Y., Marmarou, A., Brooks, D.M., and Young, H.F. (1991). Prediction tree for severely head-injured patients. J. Neurosurg. 75, 251-255.

Glorioso, C., and Sibille, E. (2011). Between destiny and disease: genetics and molecular pathways of human central nervous system aging. Prog. Neurobiol. 93, 165-181.

Heiden, J.S., Small, R., Caton, W., Weiss, M.H., and Kurze, T. (1979) Severe head injury and outcome: a prospective study. In: Neural Trauma. A.J. Popp, et al. (eds). Raven Press: New York, pps. 181-193.

Howard, M.A. III, Gross, A.S., Dacey, R.G., and Winn, H.R. (1989). Acute subdural hematomas: an age-dependent clinical entity. J. Neurosurg. 71, 858-863.

Hukkelhoven, C.W., Steyerberg, E.W., Rampen, A.J., et al. (2003). Patient age and outcome following severe traumatic brain injury: an analysis of 5600 patients. J. Neurosurg. 99, 666-673.

ISTAT. http://demo.istat.it/pop2002/index.html.

Knaus, W.A., Zimmermann, J.E., Wagner, D.P., Draper, E.A., and Lawrence, D.E. (1981). APACHE-Acute physiology and chronic health evaluation: a physiologically based classification system. Crit. Care Med. 9, 591-597.

Kovacs, E.J. (2005). Aging, traumatic injury, and estrogen treatment. Exp. Gerontol. 40, 549-555.

Lu, J., Marmarou, A., Choi, S., Maas, A., Murray, G., and Steyerberg, E.W.; IMPACT and ABIC Study Group. (2005). Mortality from traumatic brain injury. Acta Neurochir. Suppl. 95, 281-285.

Marshall, L.F., Marshall, S.B., Klauber, M.R., et al. (1991). A new classification of head injury based on computerized tomography. J. Neurosurg. 75, s14-s20.

Maas, A.I., Stocchetti, N., and Bullock, R. (2008). Moderate and severe traumatic brain injury in adults. Lancet Neurol. 7, 728-741

McGwin, G. Jr., May, A.K., Melton, S.M., Reiff, D.A., and Rue, L.W. 3rd (2001). Recurrent trauma in elderly patients. Arch. Surg. 136, 197-203.

Murray, G.D., Butcher, I., McHugh, G.S., et al. (2007). Multivariable prognostic analysis in traumatic brain injury: results from the IMPACT study. J. Neurotrauma 24, 329-337.

Mushkudiani, N.A., Engel, D.C., Steyerberg, E.W., et al. (2007). Prognostic value of demographic characteristics in traumatic brain injury: results from the IMPACT study. J. Neurotrauma 24, 259-269.

Stein, S.C., Georgoff, P., Meghan, S., Mizra, K., and Sonnad, S.S (2010). 150 years of treating severe traumatic brain injury: a systematic review of progress in mortality. J. Neurotrauma 27, 1343-1353.

Stocchetti, N., Pagan, F., Calappi, E., et al. (2004). Inaccurate early assessment of neurological severity in head injury. J. Neurotrauma 21, 1131-1140.

Stocchetti, N., Zanaboni, C., Colombo, A., et al. (2008). Refractory intracranial hypertension and "second-tier" therapies in traumatic brain injury. Intensive Care Med. 34, 461467.

Teasdale, G., and Jennett, B. (1976). Assessment and prognosis of coma after head injury. Acta Neurochir. (Wien.) 34, 4555.

Thompson, H.J., McCormick, W.C., and Kagan, S.H. (2006). Traumatic brain injury in older adults: epidemiology, outcomes, and future implications. J. Am. Geriatr. Soc. 54, 15901595. 
Tokutomi, T., Miyagi, T., Ogawa, T., et al. (2008). Age-associated increases in poor outcomes after traumatic brain injury: a report from the Japan Neurotrauma Data Bank. J. Neurotrauma $25,1407-1414$.

Vollmer, D.G., Torner, J.C., Jane, J.A., et al. (1991). Age and outcome following traumatic coma: why do older patients fare worse? J. Neurosurg. 75, s37-s49.

Wilson, J.T., Pettigrew, L.E., and Teasdale, G.M. (1998). Structured interviews for the Glasgow Outcome Scale and the Extended Glasgow Outcome Scale: guidelines for their use. J. Neurotrauma 15, 573-585.

Woolcott, J.C., Richardson, K.J., Wiens, M.O., et al. (2009). Metaanalysis of the impact of 9 medication classes on falls in elderly persons. Arch. Intern Med. 169, 1952-1960.
World Health Organization. (2010). Global status report on road safety. World Health Organization, Department of Violence \& Injury Prevention \& Disability (VIP).

Address correspondence to: Nino Stocchetti, Ph.D.

Fond. IRCCS Cà Granda Ospedale Maggiore Policlinico Neurointensive Care Unit

Pad. Monteggia

Via Francesco Sforza 35

Milan 2122 Italy

E-mail: stocchet@policinico.mi.it 